\title{
Alerts from the mental status changes of spine patients during COVID-19 outbreaks in a Grade-A Tertiary Hospital in Anhui province, China
}

\author{
Yongshun Zheng ${ }^{1}$, Xingfang Zhang ${ }^{1}$, Fan Zhang ${ }^{1}$, and Shiyuan Fang ${ }^{2}$ \\ ${ }^{1}$ First Affiliated Hospital of Anhui Medical University \\ ${ }^{2}$ Anhui Provincial Hospital
}

May 27, 2020

\begin{abstract}
Abstract Background As the coronavirus disease 2019 (COVID-19) spreads worldwide, the virus containment measures significantly affected the travel, work and live, which may bring negative impacts on the mental status of people, especially on patients. Measures This study take the spine surgery department as an example in The First Affiliated Hospital of Anhui Medical University in Hefei, Anhui, China. A psychological scale were constructed to assess the mental status of patients who were admitted to hospital during Jan 24 to Apr 2. The number of newly confirmed COVID-19 patients in Anhui, China were collected each day during Jan 24 to Apr 2. Results The mental status of patients were relatively poor at the beginning of the outbreaks and then gradually returned to normal. The trends between the psychological of patients and the number of newly confirmed COVID patients were similar. Conclusion The outbreaks adversely affected the mental state of spine patients, which showed a similar trend with the number of newly confirmed patients. However, the connection between them required further study to identify. This phenomenon may be widespread in spine surgery department and other departments, which required more attention.
\end{abstract}

Alerts from the mental status changes of spine patients during COVID-19 outbreaks in a Grade-A Tertiary Hospital in Anhui province, China

Running Title: Alerts from mental status changes during COVID-19

Yongshun Zheng ${ }^{1}$, Xingfang Zhang ${ }^{1}$, Fan Zhang ${ }^{1 *}$, Shiyuan Fang ${ }^{2 *}$

${ }^{1}$ Department of Spine Surgery, The First Affiliated Hospital of Anhui Medical University, Hefei, Anhui, China.

${ }^{2}$ Department of Orthopaedic Trauma, Affiliated Anhui Provincial Hospital of Anhui Medical University, Hefei, Anhui, China.

* Corresponding to: Fan Zhang, Department of Spine Surgery, The First Affiliated Hospital of Anhui Medical University, 81 Meishan Road, Hefei, Anhui 230032, China.

E-mail: Zhang12368258@163.com

Shiyuan Fang, Department of Orthopaedic Trauma, Affiliated Anhui Provincial Hospital of Anhui Medical University, 17 Lujiang Road, Hefei, Anhui, 230001, China.

E-mail: Fang289669676@163.com

\section{Keywords}


COVID-19, spine surgery department, psychology, emergency response

\begin{abstract}
Background As the coronavirus disease 2019 (COVID-19) spreads worldwide, the virus containment measures significantly affected the travel, work and life, which may bring negative impacts on the mental status of people, especially on patients.

Measures This study take the spine surgery department as an example in a Grade-A tertiary hospital in Anhui province, China. A psychological scale were constructed to assess the mental status of patients who were admitted to hospital during Jan 24 to Apr 2. The number of newly confirmed COVID-19 patients in Anhui, China were collected each day during Jan 24 to Apr 2.

Results The mental status of patients were relatively poor at the beginning of the outbreaks and then gradually returned to normal. The trends between the psychological of patients and the number of newly confirmed COVID patients were similar.
\end{abstract}

Conclusion The outbreaks adversely affected the mental state of spine patients, which showed a similar trend with the number of newly confirmed patients. However, the connection between them required further study to identify. This phenomenon may be widespread in spine surgery department and other departments, which required more attention.

\title{
1. Introduction
}

The emergence of Coronavirus Disease 2019 (COVID-19) and its subsequent spread has lived up to many warnings and caused an evolving global public health crisis(Hartley \& Perencevich, 2020). Current studies reported that the outbreaks and trauma brought multiple new stresses, including physical and psychological health risks, isolation and loneliness, the closure of many schools and businesses, economic vulnerability and job losses, and even the domestic violence(Bradbury-Jones \& Isham, 2020; Cukor et al., 2011; Paakkari \& Okan, 2020). According to data of Chinese Center for Disease Control and Prevention, Anhui declared first-level emergency response (ER) on Jan 24 and down-regulated the level on Feb 25 and Mar 15. The ER means that the movement, transportation and business were restricted and during the first-level ER, only emergency patients were received in hospital(Munster, Koopmans, van Doremalen, van Riel, \& de Wit, 2020). The present study aimed to investigate the mental status changes of spine patients during COVID-19 outbreaks and provide timely alerts.

\section{Methods}

The patients in spine surgery department in a Grade-A tertiary hospital in Anhui province, China from Jan 24 to Apr 2, 2020 were recorded. The psychological scale (Table 1) were constructed and the total scores ranged from 0 to 12(Bradbury-Jones \& Isham, 2020; Cukor et al., 2011; Paakkari \& Okan, 2020). Patients were asked to finish the scale during Jan 24 to Apr 2. The number of the newly confirmed COVID-19 patients each day in Anhui, China were collected from Jan 24 to Apr 2. All statistical analyses were performed with GraphPad Prism 6.02 software.

\section{Results}

The results showed that the psychological scores of patients increased at first and then decreased, which means their mental status were poor at the early stage of the outbreaks and then returned to normal gradually, but the scores were still around 2 (Figure 1). Similarly, the number of newly confirmed patients first increased and then decreased in Anhui (Figure 1).

\section{Discussion}

During COVID-19 outbreaks, the ER effectively cut off the virus spread in Anhui. However, the quarantine and trauma related psychological distress should not be ignored, which may adversely affect the course of the disease, the treatment process, and reduce quality of life(Brooks et al., 2020; Cukor et al., 2011; Jarosz, Syed, Blachut, \& Badura Brzoza, 2020). The trend of mental status may due to normal panic at the beginning of 
the outbreaks and then gradually adapted to the situation. However, the scores not fell to zero, which may be attributed the constant worry about the outbreaks reappear or just nervous due to their spine diseases. Therefore, more humanistic approaches are required, including solving their problems in life, sending positive and optimistic attitude towards life and reducing their fear on COVID-19. However, It was important to attach importance to the preventive measures on COVID-19 while relieve their panic. Notably, the figures of psychological scores and newly confirmed patients showed similar trends, but the connection between them required farther research to verified. In the present study, we took the spine surgery department as an example to investigate the mental status of patients, yet patients in other departments may encounter a similar situation. Meanwhile, China has got the virus fully under control while the outbreaks in many other countries is becoming much worse, which means the potential impacts on the mental status of patients cannot be ignored. All of these alerts required further studies to verified.

Figure Legend

Figure 1: The average total points and the number of newly confirmed patients varied with the date

The total scores of each patients were collected based on the psychological scale from Jan 24 to Apr 2. Results were presented as mean \pm SD. There were no patients for a few days and the mean \pm SD value were blank on that day and the SD were blank if the patient were only one on that day. The number of the newly confirmed patients each day from Jan 24 to Apr 2 in Anhui, China were collected. The two lines are distinguished by a solid line and a dashed line.

\section{Acknowledgements}

The authors thank all the participants and the nursing team of The First Affiliated Hospital of Anhui Medical University who helped to collect and organize the data

\section{Ethical Statement}

Ethical Statement is not applicable

\section{Competing Interests}

Authors declared no competing interests, financial or otherwise

\section{Data Availability Statement}

The data that support the findings of this study are available from the corresponding author upon reasonable request.

\section{Reference}

Bradbury-Jones, C., \& Isham, L. (2020). The pandemic paradox: the consequences of COVID-19 on domestic violence. J Clin Nurs . doi: 10.1111/jocn.15296

Brooks, S. K., Webster, R. K., Smith, L. E., Woodland, L., Wessely, S., Greenberg, N., \& Rubin, G. J. (2020). The psychological impact of quarantine and how to reduce it: rapid review of the evidence. Lancet, 395 (10227), 912-920. doi: 10.1016/S0140-6736(20)30460-8

Cukor, J., Wyka, K., Jayasinghe, N., Weathers, F., Giosan, C., Leck, P., . . . Difede, J. (2011). Prevalence and predictors of posttraumatic stress symptoms in utility workers deployed to the World Trade Center following the attacks of September 11, 2001. Depress Anxiety, 28 (3), 210-217. doi: 10.1002/da.20776

Hartley, D. M., \& Perencevich, E. N. (2020). Public Health Interventions for COVID-19: Emerging Evidence and Implications for an Evolving Public Health Crisis. JAMA . doi: 10.1001/jama.2020.5910

Jarosz, M., Syed, S., Blachut, M., \& Badura Brzoza, K. (2020). Emotional distress and quality of life in allergic diseases. Wiad Lek, 73 (2), 370-373. 
Munster, V. J., Koopmans, M., van Doremalen, N., van Riel, D., \& de Wit, E. (2020). A Novel Coronavirus Emerging in China - Key Questions for Impact Assessment. N Engl J Med, 382 (8), 692-694. doi: 10.1056/NEJMp2000929

Paakkari, Leena, \& Okan, Orkan. (2020). COVID-19: health literacy is an underestimated problem. The Lancet Public Health . doi: 10.1016/s2468-2667(20)30086-4

\section{Hosted file}

Table 1.docx available at https://authorea.com/users/326932/articles/454623-alerts-from-themental-status-changes-of-spine-patients-during-covid-19-outbreaks-in-a-grade-a-tertiaryhospital-in-anhui-province-china

\section{Figure 1}

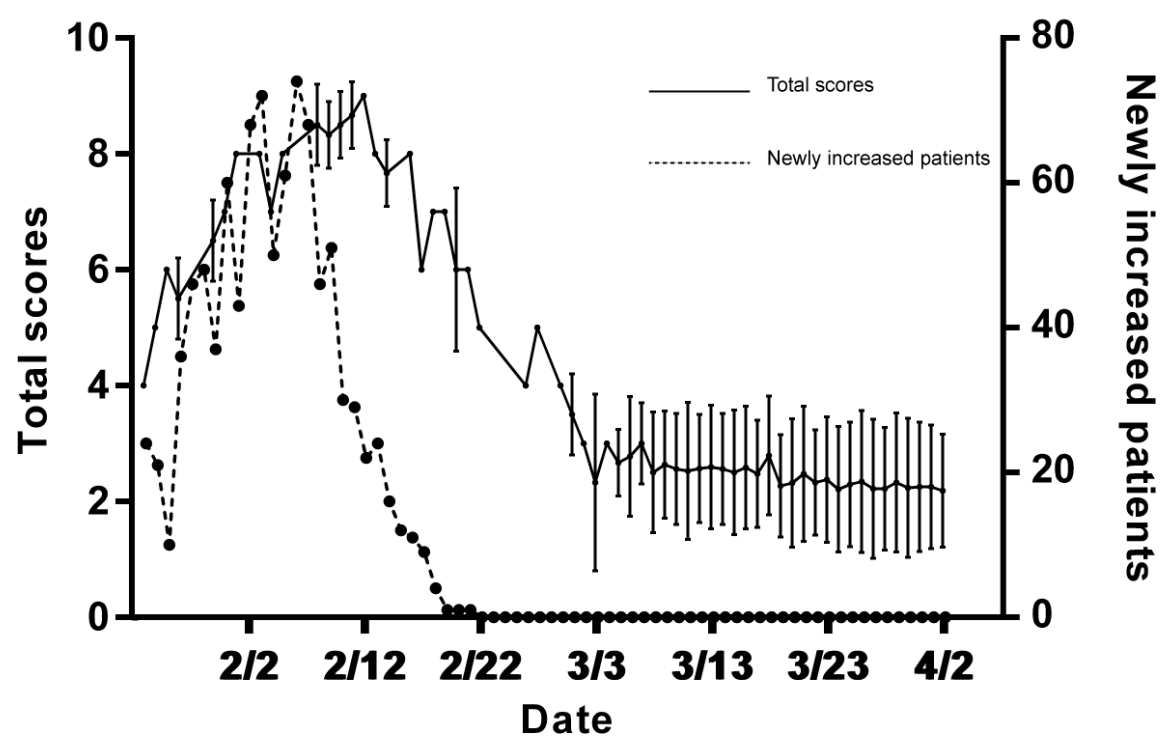

